This item was submitted to Loughborough's Research Repository by the author.

Items in Figshare are protected by copyright, with all rights reserved, unless otherwise indicated.

\title{
Acoustic band gap formation in metamaterials
}

PLEASE CITE THE PUBLISHED VERSION

http://dx.doi.org/10.1142/S0217979210057110

\section{PUBLISHER}

(c) World Scientific Publishing Company

VERSION

AM (Accepted Manuscript)

LICENCE

CC BY-NC-ND 4.0

\section{REPOSITORY RECORD}

Elford, Daniel P., Luke Chalmers, F.V. Kusmartsev, and G.M. Swallowe. 2019. "Acoustic Band Gap Formation in Metamaterials". figshare. https://hdl.handle.net/2134/7985. 
This item was submitted to Loughborough's Institutional Repository (https://dspace.lboro.ac.uk/) by the author and is made available under the following Creative Commons Licence conditions.

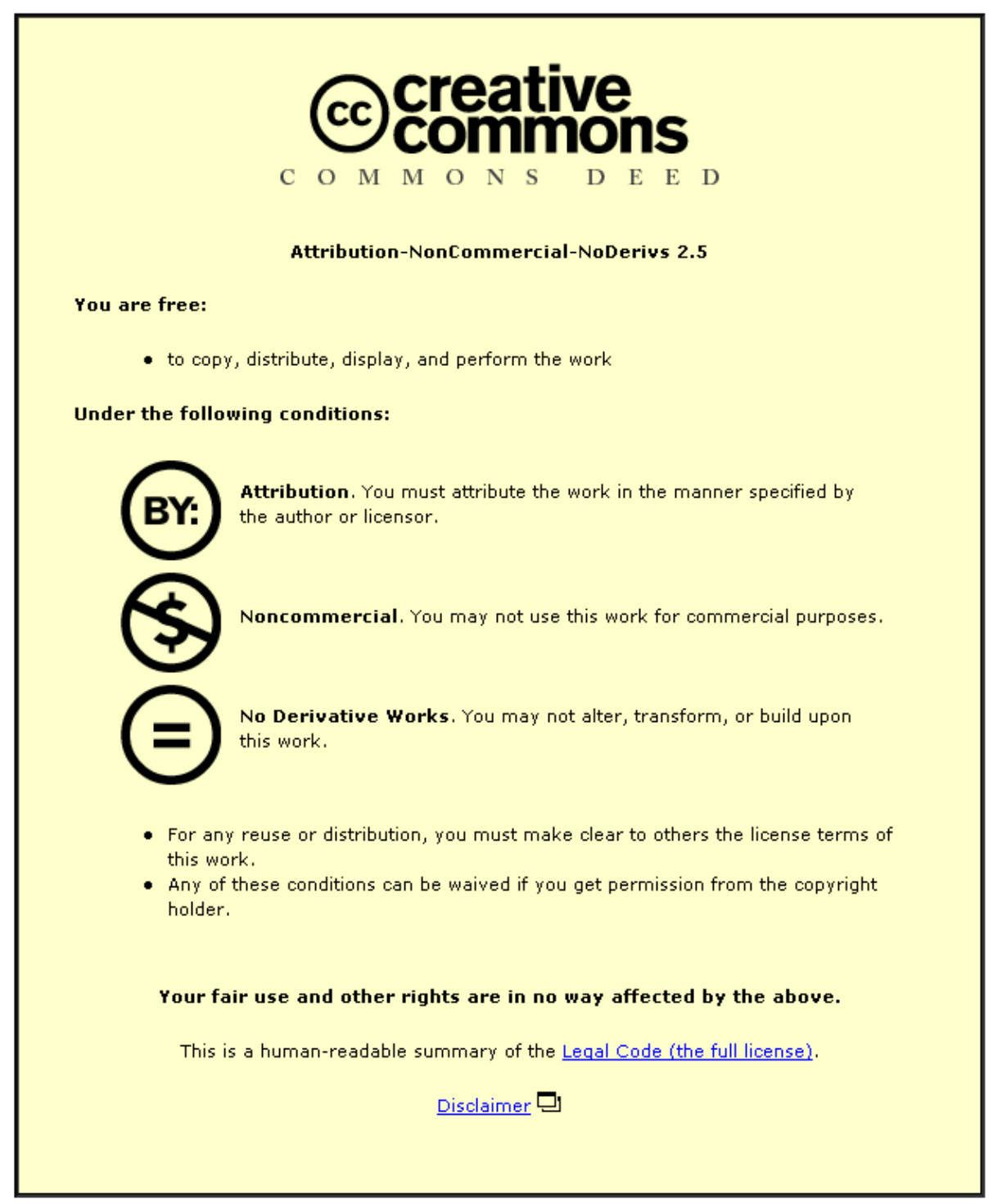

For the full text of this licence, please go to: http://creativecommons.org/licenses/by-nc-nd/2.5/ 


\title{
Acoustic Band Gap Formation in Metamaterials
}

\author{
D.P. Elford, L. Chalmers, F. Kusmartsev* and G.M. Swallowe \\ Department of Physics, Loughborough University, Loughborough LE11 3TU, United Kingdom \\ *E-mail: F.kusmartsev@lboro.ac.uk
}

\begin{abstract}
We present several new classes of metamaterials and/or locally resonant sonic crystal that are comprised of complex resonators. The proposed systems consist of multiple resonating inclusion that correspond to different excitation frequencies. This causes the formation of multiple overlapped resonance band gaps. We demonstrate theoretically and experimentally that the individual band gaps achieved, span a far greater range $(\approx 2 \mathrm{kHz})$ than previously reported cases. The position and width of the band gap is independent of the crystal's lattice constant and forms in the low frequency regime significantly below the conventional Bragg band gap. The broad envelope of individual resonance band gaps is attractive for sound proofing applications and furthermore the devices can be tailored to attenuate lower or higher frequency ranges, i.e. from seismic to ultrasonic.
\end{abstract}

Keywords: Acoustic metamaterials; sonic crystals; resonance band gaps

\section{Introduction}

Acoustic wave propagation in sonic crystals (SC's) has been studied since the late 1980 's ${ }^{1}{ }^{2}$ Such structures forbid the transmission of sound in a band gap when its wavelength is comparable to the periodic spacing of the scattering units..$^{3-9}$ As such, Bragg's law is satisfied and a band gap is formed (Bragg band gap - BBG). This condition imposes a restriction in designing conventional SC's as the lattice spacing of the array is intrinsically linked to the attenuated frequency range. Subsequently a new class of SC was proposed, known as a locally resonant sonic material (LRSM), ${ }^{10}$ which form resonance band gaps (RBG) that occur at frequencies independent of the structures lattice constant. A further extension upon this concept utilised several resonators to form multiple RBG's that can be overlapped. ${ }^{11}$

We present here locally resonant sonic crystal (LRSC) systems, which are comprised of two classes of complex resonators, being analogous to Helmholtz and quarter-wave resonators respectively. Also detailed is a third LRSC system with resonators that have a geometry similar to Nautilus shells, that combine aspects from the first two designs. All systems have been found to exhibit multiple broad band gaps, in the lower frequency range below Bragg gap formation. We show that the position and width of the RBG's can be tuned by adjusting the resonator properties, such that, multiple band gaps can be combined to form broad regions of frequency attenuation. This can be exploited to create band gaps of any desired 
width. The acoustic resonators described here operate with a low quality (Q) factor and form RBG's much wider than previously reported. ${ }^{11}$

To investigate the RBG formation experimentally in the first LRSC system, Cshaped resonators were constructed by machining a single $4 \mathrm{~mm}$ wide longitudinal slot along the length of a steel tube, with external diameter $13 \mathrm{~mm}$, internal diameter $9.7 \mathrm{~mm}$ and length $300 \mathrm{~mm}$. The LRSC was constructed from an array of such resonators, positioned in a $10 \times 10$ square configuration with lattice constant $22 \mathrm{~mm}$. The length of the scatterers was sufficiently longer than the relevant wavelength range being tested ensuring that the environment can be assumed to be quasi-twodimensional. To measure attenuation levels a 150W Realistic Nova 800 speaker with a maximum frequency response of $20 \mathrm{kHz}$ was driven by a Bruel \& Kjaer beat frequency oscillator (type 1022), which was in turn driven by a Bruel \& Kjaer level recorder (type 2305). This allowed a six-second rising tone sample from 1-10 kHz to be generated. The signal was transmitted through the LRSC systems and recorded using a dual Behringer C-2 studio condenser microphone setup. Control recordings were obtained by performing the experiment without a crystal present. A frequency spectrum was obtained by performing a Fourier analysis of the recording and sound pressure levels were calculated from the relative amplitudes of the control and LRSC system being tested. The frequency spectra were then compared to locate regions of attenuation. All acoustic experiments were performed inside an anechoic chamber.

\section{Numerical Simulations of the Sound Propagation through the Resonance Media}

In order to interpret the experimental results extensive finite element (FE) simulations were performed modelling acoustic wave propagation and scattering through LRSC's comprised of C-shaped resonators. A time harmonic analysis of the acoustic wave propagation was performed using the acoustics package of the FE software COMSOL Multiphysics v3.4a. ${ }^{12}$ For a time harmonic pressure wave excitation of the form $p=p_{0}(x, y) e^{i \omega t}$, the wave equation reduces to the Helmholtz equation, which is used to describe the acoustic wave propagation:

$$
\nabla \cdot\left(-\frac{1}{\rho_{0}} \nabla p_{0}\right)-\frac{\omega^{2} p_{0}}{\rho_{0} c^{2}}=0
$$

Where $p_{0}$ is the pressure, depending on $x$ and $y$ spatial dimensions, $c$ is the speed of sound, $\rho_{0}$ is the density and $\omega=2 \pi f$ is the angular frequency. By solving this equation inside the domain we obtain the resulting pressure field. The resonating units in the LRSC system consist of steel tubes that are modelled as fluid inclusions with a high stiffness and mass embedded in air. Therefore, the high contrast between the steel and air parameters gives rise to a high reflection coefficient, even though a small pressure field may be present inside the resonators walls.

The transmitted acoustic frequency spectrum obtained from the FE simulation for the C-shaped resonator LRSC shows the appearance of two distinct band gaps 
as seen in Fig.1.(A). It is possible to distinguish between the BBG and RBG mechanisms by comparison with a spectrum obtained from a conventional SC of the same size but with non-resonating inclusions. This structure forms a BBG but does not demonstrate any local resonance characteristics and hence, no RBG is formed. The BBG is located at $8.15 \mathrm{kHz}$, as predicted by Bragg's law, and spans from 6.9-9.4 $\mathrm{kHz}$. This is in good agreement with the experimentally obtained band gap shown in Fig.1.(B), which forms from $6.8-9.8 \mathrm{kHz}$, centered at $8.3 \mathrm{kHz}$. The simulation shows the C-shaped cavities acting analogously to Helmholtz resonators that have a maximum response at their excitation frequency, of $4.7 \mathrm{kHz}$ and form a $\mathrm{RBG}$ centered at this frequency spanning from 3.9-5.5 kHz. The experimentally obtained spectrum confirms the appearance of this gap from 3.8-6.1 kHz. Attenuation levels achieved in the experimental results reach a maximum at $16 \mathrm{~dB}$ and $20 \mathrm{~dB}$ for the BBG and RBG respectively. This is a suitably attractive level of attenuation for soundproofing applications, as a $9 \mathrm{~dB}$ decrease would remove $80 \%$ of unwanted noise.

Each C-shaped resonator within the LRSC acts as an individual Helmholtz resonator, with a broad neck, containing a fixed volume of air. The broad neck induces the formation of broad band excitations in the resonators. When a pressure variation, in the form of a sound wave, interacts with the air in the neck, the pressure of the air inside increases. As the external force is removed, the pressure equalizes and forces air back through the neck. Due to the inertia of the air in the neck, a region of low pressure is created in the cavity, which in turn causes air to be drawn back in. The air then continues to oscillate at frequency $\omega_{0}$, which is dependent upon the characteristic dimensions of the resonators cavity and neck, but is shape and material independent. When an incoming wave of angular frequency $\omega$ is incident upon the resonating unit with excitation frequency $\omega_{0}$ the linear response function is proportional to $1 /\left(\omega_{0}^{2}-\omega^{2}\right)$ causing exponential attenuation of the wave. ${ }^{10} \mathrm{At}$ frequencies below Bragg's condition and away from resonance, the LRSC systems present a homogeneous structure to the sound waves. The resonant frequency $f_{0}$ of the C-shaped resonating units may be tuned using an empirically obtained equation derived from Helmholtz's equation for resonators.

$$
f_{0}=\frac{c}{\pi^{5 / 4}} \frac{\sigma^{1 / 4}}{S^{1 / 2}}
$$

Where $\sigma$ is the slot width and $S=\pi r^{2}$ for our two-dimensional scenario. We can assume from this equation that when the slot width is increased for a fixed diameter resonator, the frequencies should increase by a factor of $\sigma^{1 / 4}$ and for a constant slot width, the frequencies should decrease inversely proportionally to the diameter.

\section{Experiments and Comparison with the Theory}

To validate the existence of the RBG mechanism, experiments were conducted upon a random distribution of C-shaped resonators, see Fig.1.(B). Due to the lack of periodicity in this structure, the array is disordered and BBG formation cannot occur. 

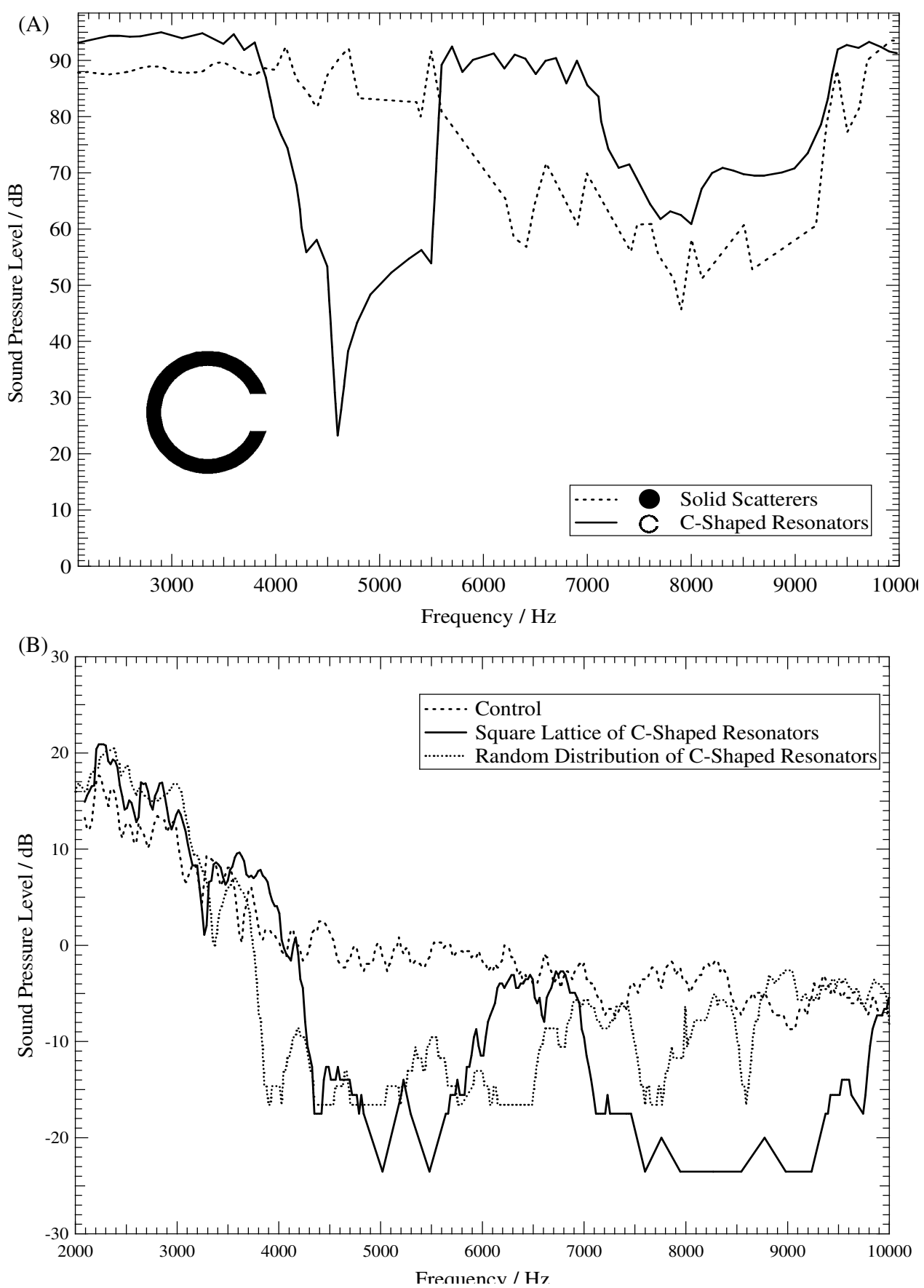

Fig. 1. (A) Frequency spectrum obtained by FE simulation of a LRSC consisting of C-shaped resonator units (solid line). Included is a frequency spectrum obtained for a conventional sonic crystal comprised of solid scattering units for comparison (dashed line). (B) Experimentally obtained frequency spectrum of a LRSC consisting of C-shaped resonators as described in Fig.1.(A) (solid line). Included on the plot are spectra for a control sample (dashed line) and for a LRSC with a random distribution of C-shaped resonators (dotted line). 
However, the RBG is still observed due to its independence from the arrays periodicity. Each C-shaped resonator acts independently and accounts for a small amount of absorption, that when summed for the whole system, gives appreciable levels of sound attenuation forming a RBG for this disordered system. Similar measurements were performed using the same Bruel \& Kjaer system as detailed above. The experimentally obtained frequency spectrum shows the existence of an RBG from 3.5-6.5 $\mathrm{kHz}$.

In order to enlarge the wavelength range in which the LRSC is active, a multiple RBG system is simulated using several C-shaped resonators of varying dimensions in a mixed array configuration. In such an arrangement, several RBG's are formed, and by careful selection of the cavity dimensions, these individual gaps can be overlapped to form a combined broader gap. The frequency spectrum, see Fig.2.(A), shows RBG's for $11 \mathrm{~mm}$ and $14 \mathrm{~mm}$ diameter resonators, from $5.5-7.5 \mathrm{kHz}$ with $65 \mathrm{~dB}$ of attenuation at its peak and at $4.8-6.0 \mathrm{kHz}$ with $60 \mathrm{~dB}$ of attenuation. A frequency spectrum obtained from a mixed LRSC with alternating layers shows the formation of a combined band gap system enveloping the two individual resonance peaks, 5.0$7.5 \mathrm{kHz}$ with similar levels of attenuation. In effect, the width of the band gap is doubled using two carefully selected resonator sizes. Furthermore, the RBG can be widened with more than two resonator sizes in a single array, thus allowing band gaps of any width to be created.

\section{Acoustic Metamaterials made of the Matryoshka Resonators}

We propose an alternative design to this basic mixed system that is comprised of an arrangement of concentric resonators coined the Matryoshka (Russian doll) configuration, see insert on Fig.2.(B). The inclusion of multiple sized resonating units gives rise to the formation of many RBG's with substantial levels of attenuation, and the concentric distribution gives an overall reduction in array size. The frequency spectrum for a Matryoshka array comprised of $11 \mathrm{~mm}$ and $14 \mathrm{~mm}$ diameter resonators forms two individual RBG's from $5.6-6.5 \mathrm{kHz}$ and $3.5-5.1 \mathrm{kHz}$ respectively, see Fig.2.(B). These RBG's can again be overlapped, by careful selection of the resonator dimensions, allowing LRSC systems to be created with very broad frequency ranges. The compactness of the Matryoshka configuration allows an acoustic barrier to be constructed from a single layer of resonators, whilst still offering reasonable levels of attenuation.

An alternative LRSC system is proposed that utilises a second class of acoustic resonator inclusions and is again able to form an enlarged RBG by overlapping resonances. The inclusions are quarter wave resonators that have a fundamental excitation frequency $f_{0}$, which is active when the length of the resonator is a quarter of a wavelength long. Harmonics of this fundamental frequency also exist at integer multiples of $f_{0}$. As an acoustic wave is incident upon the resonator, part of the wave enters the cavity and is reflected back. In the time, the acoustic wave takes to travel down the resonator and back to the opening, the acoustic wave outside of the 

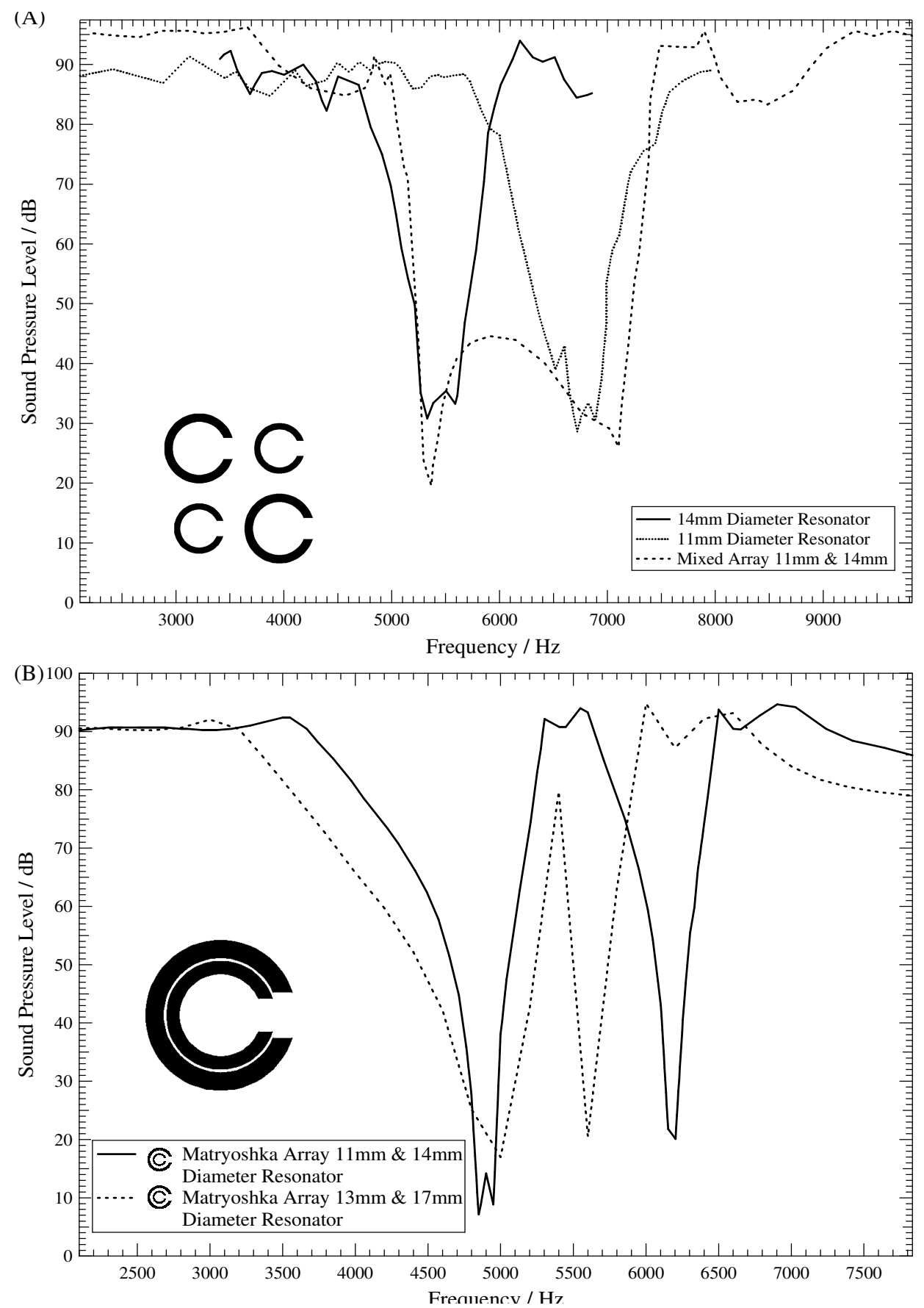

Fig. 2. (A) FE calculated frequency spectrum for a LRSC comprised of two different diameter C-shaped resonators, $14 \mathrm{~mm}$ and $11 \mathrm{~mm}$, (dashed line) indicating the presence of a RBG envelope. Included on the plot are the frequency spectra for LRSC's comprised of each of the individual sized resonators for comparison. (B) Frequency spectrum for a LRSC with Matryoshka (Russian doll) inclusions, comprised of $11 \mathrm{~mm}$ and $14 \mathrm{~mm}$ C-shaped resonators. 

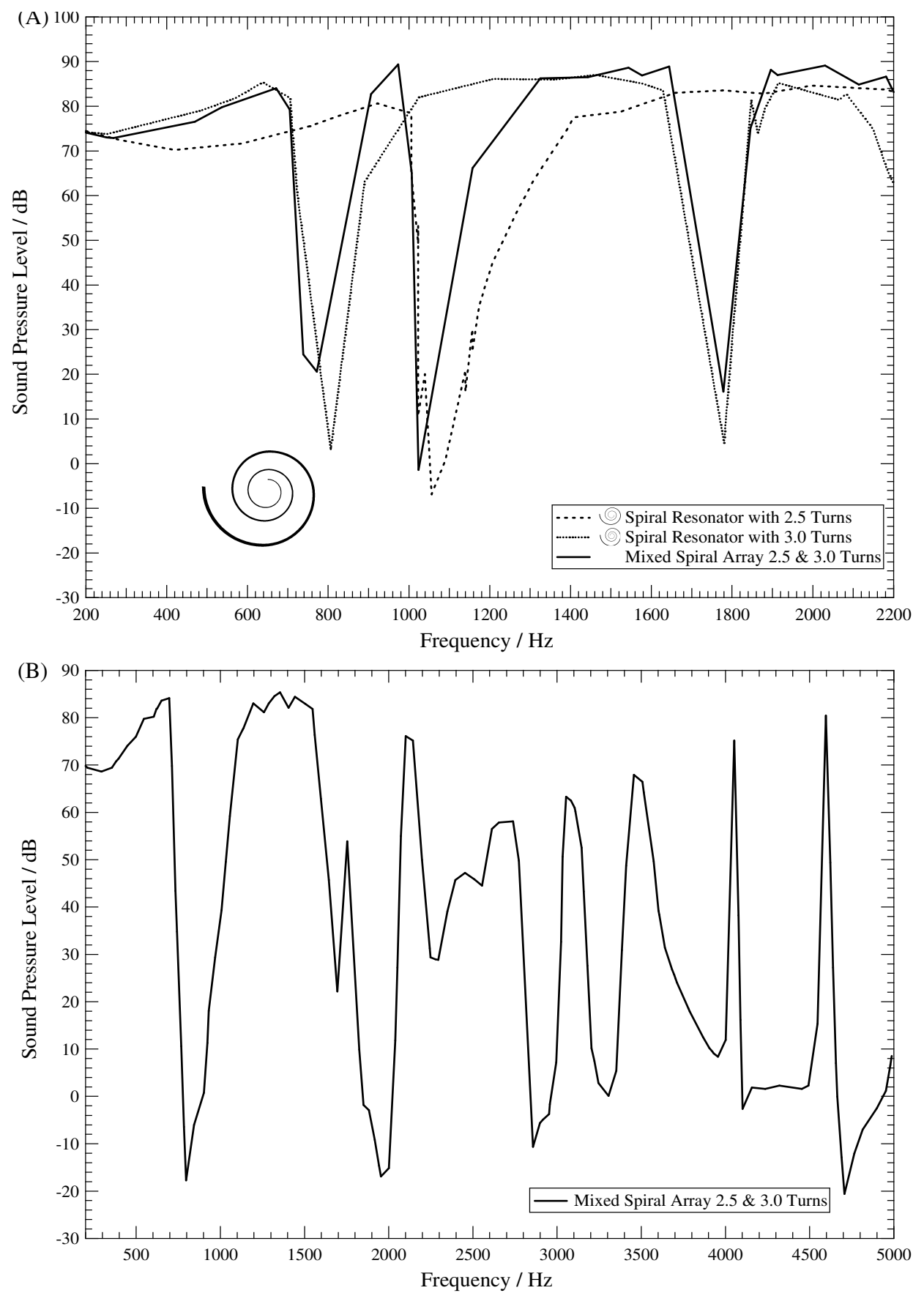

Fig. 3. (A) Frequency spectra obtained from FE simulations for a LRSC comprised of spiral resonators with 2.5 turns (dashed line), 3.0 turns (dotted line) and a mixed configuration (solid line). The spectra are shown over the low frequency regime $0.2-2.2 \mathrm{kHz}$ to detail the overlapping mechanism. (B) An extended frequency spectrum ranging from 0-5 kHz for the LRSC with the mixed spiral inclusions which, shows an envelope of multiple RBG's corresponding to the fundamental and harmonic excitation frequencies of each resonating unit. 
resonator has shifted half a wavelength, and the two waves interfere destructively causing attenuation. As the length of the resonator is intrinsically linked to the excitation frequency, the characteristic length of such resonators needed to attenuate low frequency noise is approximately $0.5 \mathrm{~m}$ for $150 \mathrm{~Hz}$. This is an undesirable scale for sound proofing applications and therefore the length of the resonator can be coiled into a more compact spiral design, reducing the resonator size to $\mathrm{mm}$ scale. The spiral resonator schematic is inset on Fig.3.(A). This geometry allows the formation of quarter wave resonating units that are active at wavelengths significantly larger than their size.

FE simulations have been performed on a 2D LRSC comprised of such spiral resonators. The first unit used was a Bernoulli type spiral with external radius $0.0128 \mathrm{~m}$ and decay per $90^{\circ}$ of $86 \%$ with 3.0 turns. This spiral has a characteristic path length of $0.16 \mathrm{~m}$ and a corresponding fundamental frequency $f_{0}=0.74 \mathrm{kHz}$ as given by quarter wave resonator theory. ${ }^{13}$ When included in a LRSC system a fundamental RBG is observed from $0.68-0.9 \mathrm{kHz}$ with $60 \mathrm{~dB}$ of attenuation, see Fig.3.(A). A higher order harmonic also exists at double the fundamental frequency at $1.72 \mathrm{kHz}$ with similar levels of attenuation. A second spiral with external radius $0.0103 \mathrm{~m}$ and the same decay, but with only 2.5 turns is also included. This resonator unit has a shorter path length of $0.11 \mathrm{~m}$, and hence a higher fundamental excitation frequency $f_{0}=1.05 \mathrm{kHz}$. The frequency spectrum demonstrates this with the appearance of a RBG from $0.96-1.38 \mathrm{kHz}$ with attenuation peaking at $70 \mathrm{~dB}$. A LRSC comprised of a mixed array of the 2.5 and 3.0 turn Bernoulli spiral resonators was then simulated and demonstrates combined resonances as with the mixed array of C-shaped resonators. Similarly, the band gap envelope covers the individual resonances of each unit. The overlapping of the fundamental RBG's also causes combined RBG formation in the harmonic bands. This premise has been exploited to form a LRSC with multiple overlapping fundamental and harmonic RBG's, see Fig.3.(B).

\section{Media Made of the 'Seashell' Resonators}

A final LRSC system was modelled that again exhibits a large number of broad local resonances. It is comprised from a third class of acoustic resonator unit, coined the 'seashell' resonator. The design of this unit incorporates aspects of each of the previous geometries, namely the inclusion of multiple sized resonating cavities from the Matryoshka resonator and the space saving feature of the spiral resonator. The appearance of this system resembles that of one found in nature, namely the Nautilus shell, see Fig.4.(A). It has a spiral profile with logarithmic progression and is divided into several separate chambers, each section of which is connected by a small opening. This system is analogous to our seashell resonators, which are a collection of individual resonating cavities, with different excitation frequencies. Therefore, both examples should have a similar acoustic response and form a large number of broad resonances spanning over a large frequency range. For a Nautilus shell, the 
amplification of background noise over a broad range of frequencies produces pink noise. This is in fact what is commonly mistaken to be sound of the sea when a shell is placed next to one's ear.

FE simulations have been performed for a LRSC comprised of seashell resonators with eight resonant cavities, and its frequency response is presented in Fig.4.(B). This system demonstrates multiple RBG's, corresponding to each of the cavities dimensions, that span a broad range of frequencies from $10 \mathrm{~Hz}-20 \mathrm{kHz}$. The excitation frequencies of the cavities lie very close to each other and thus, due to the large width of each RBG, an enlarged band gap envelope is formed with an average level of attenuation of approximately 30dB. Again, a band gap of any desired width can be created by including more cavities in the seashell resonator. 


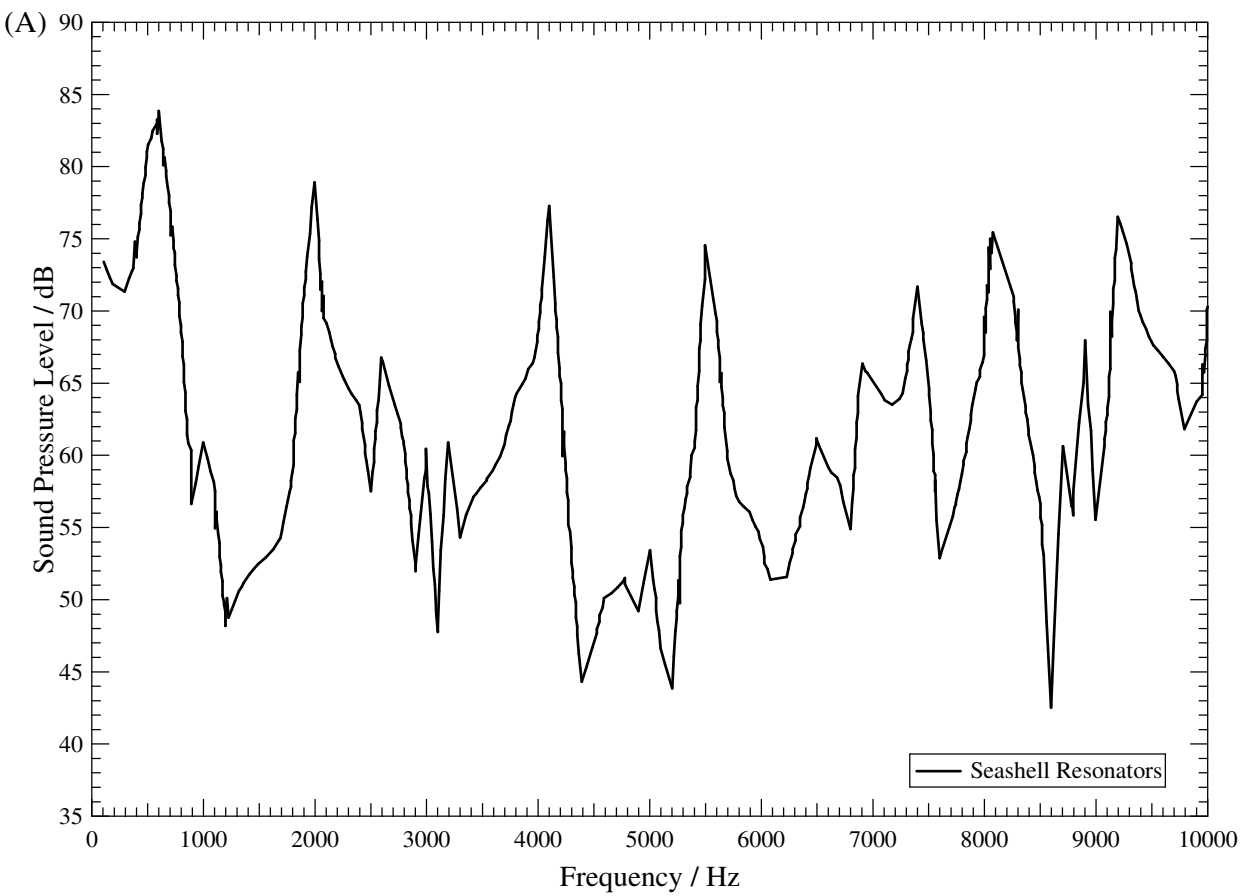

(B)
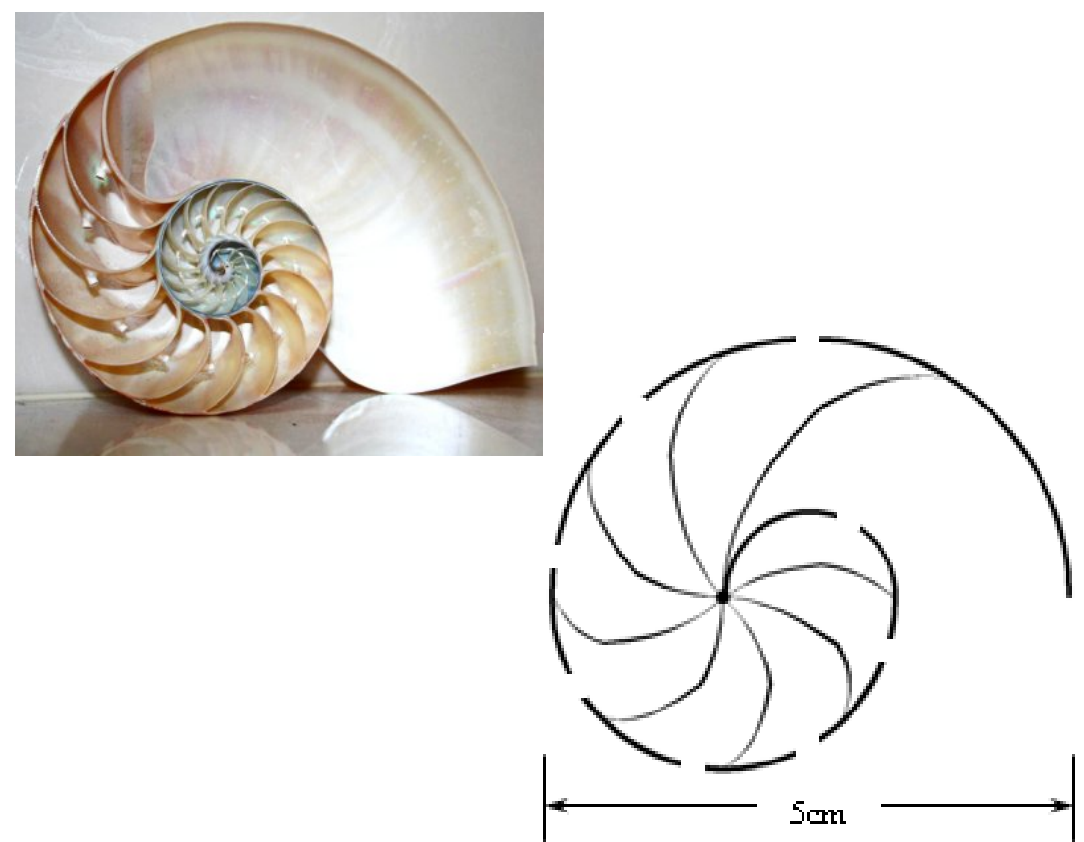

Fig. 4. (A) Frequency spectrum obtained from FE simulations for a LRSC comprised of seashell resonators with eight chambers. (B) Nature's example of a broad band resonator, in the form of a Nautilus shell, and a schematic of the similar seashell resonators used in the FE investigation. 


\section{Conclusion}

In summary, LRSC's constructed from the proposed complex resonators exhibit broad regions of acoustic attenuation, which arise due to the overlapping of several close resonance band gaps. This is most easily achieved for the C-shaped and spiral resonating system by using a simple mixed configuration of multiple sized resonators. The proposed Matryoshka and seashell LRSC systems do not require this arrangement as each unit exhibits multiple resonances itself and thus, is independent of lattice spacing. This reduces the overall size of the LRSC and allows reasonable levels of attenuation to be achieved from a single layer of resonators. Furthermore, the attenuated frequency range can be tailored by adjusting the dimensions, and hence excitation frequencies, of the resonating cavities. This can be exploited to form band gaps over lower and higher frequency regimes, i.e. seismic to ultrasonic.

\section{ackknowledgements}

The work was supported by the ESF network-program AQDJJ

\section{References}

1. Lakhtakia, A., Varadan, V.V. \& Varadan, V.K., J. Acoust. Soc. Am. 83, 1267 (1988).

2. Economou, E.N. \& Zdetsis, A., Phys. Rev. B. 40, 1334 (1989).

3. Kushwaha, M.S., Halevi, P., Dobrzynski, L. \& Djafari-Rouhani, B., Phys. Rev. Lett. 71, 2022 (1993).

4. Chen, Y.Y. \& Ye, Z., Phys. Rev. Lett. 87, 184301 (2001).

5. Chen, Y.Y. \& Ye, Z., Phys. Rev. E. 64, 036616 (2001).

6. Larabi, H., Pennec, Y., Djafari-Rouhani, B. \& Vasseur, J.O., Journal of Physics: Conference Series. 92, 012112 (2007).

7. Larabi, H., Pennec, Y., Djafari-Rouhani, B. \& Vasseur, J.O., Phys. Rev. E. 75, 066601 (2007).

8. Vasseur, J.O., et al., Phys. Rev. E. 65, 056608 (2002).

9. Sanchez-Perez, J.V., et al, Phys. Rev. Lett. 80, 5325 (1998).

10. Liu, Z., et al., Science. 289, 1734 (2000).

11. Ho, K.M., Cheng, C.K., Yang, Z, Zhang, X.X. \& Sheng, P., Appl. Phys. Lett. 83, 5566 (2003).

12. COMSOL Inc, COMSOL Multiphysics v3.4a. (2007).

13. Kinsler, L.E., Frey, A.R., Coppens, A.B. \& Sanders, J.V., Fundamentals of Acoustics, Fourth Edition, Wiley \& Sons, p274 (2000). 